\title{
Laminoplasty versus laminectomy with posterior spinal fusion for multilevel cervical spondylotic myelopathy: influence of cervical alignment on outcomes
}

\author{
Darryl Lau, MD, ${ }^{1}$ Ethan A. Winkler, MD, PhD, ${ }^{1}$ Khoi D. Than, MD, ${ }^{2}$ Dean Chou, MD, ${ }^{1}$ and \\ Praveen V. Mummaneni, MD ${ }^{1}$
}

1Department of Neurological Surgery, University of California, San Francisco; and 'Department of Neurosurgery, Oregon Health Science University, Portland, Oregon

OBJECTIVE Cervical curvature is an important factor when deciding between laminoplasty and laminectomy with posterior spinal fusion (LPSF) for cervical spondylotic myelopathy (CSM). This study compares outcomes following laminoplasty and LPSF in patients with matched postoperative cervical lordosis.

METHODS Adults undergoing laminoplasty or LPSF for cervical CSM from 2011 to 2014 were identified. Matched cohorts were obtained by excluding LPSF patients with postoperative cervical Cobb angles outside the range of laminoplasty patients. Clinical outcomes and radiographic results were compared. A subgroup analysis of patients with and without preoperative pain was performed, and the effects of cervical curvature on pain outcomes were examined.

RESULTS A total of 145 patients were included: 101 who underwent laminoplasty and 44 who underwent LPSF. Preoperative Nurick scale score, pain incidence, and visual analog scale (VAS) neck pain scores were similar between the two groups. Patients who underwent LPSF had significantly less preoperative cervical lordosis $\left(5.8^{\circ}\right.$ vs $\left.10.9^{\circ}, p=0.018\right)$. Preoperative and postoperative C2-7 sagittal vertical axis (SVA) and T-1 slope were similar between the two groups. Laminoplasty cases were associated with less blood loss (196.6 vs $325.0 \mathrm{ml}, \mathrm{p}<0.001$ ) and trended toward shorter hospital stays ( 3.5 vs 4.3 days, $p=0.054$ ). The perioperative complication rate was $8.3 \%$; there was no significant difference between the groups. LPSF was associated with a higher long-term complication rate $(11.6 \% \mathrm{vs} 2.2 \%, p=0.036)$, with pseudarthrosis accounting for 3 of 5 complications in the LPSF group. Follow-up cervical Cobb angle was similar between the groups $\left(8.8^{\circ}\right.$ vs $\left.7.1^{\circ}, p=0.454\right)$. At final follow-up, LPSF had a significantly lower mean Nurick score $(0.9$ vs $1.4, p=0.014)$. Among patients with preoperative neck pain, pain incidence $(36.4 \%$ vs $31.3 \%, p=0.629)$ and VAS neck pain ( 2.1 vs $1.8, p=0.731$ ) were similar between the groups. Similarly, in patients without preoperative pain, there was no significant difference in pain incidence $(19.4 \%$ vs $18.2 \%, p=0.926)$ and VAS neck pain $(1.0$ vs $1.1, p=0.908)$. For laminoplasty, there was a significant trend for lower pain incidence $(p=0.010)$ and VAS neck pain $(p=0.004)$ with greater cervical lordosis, especially when greater than $20^{\circ}(p=0.011$ and $p=0.018)$. Mean follow-up was 17.3 months.

CONCLUSIONS For patients with CSM, LPSF was associated with slightly greater blood loss and a higher long-term complication rate, but offered greater neurological improvement than laminoplasty. In cohorts of matched follow-up cervical sagittal alignment, pain outcomes were similar between laminoplasty and LPSF patients. However, among laminoplasty patients, greater cervical lordosis was associated with better pain outcomes, especially for lordosis greater than $20^{\circ}$. Cervical curvature (lordosis) should be considered as an important factor in pain outcomes following posterior decompression for multilevel CSM.

https://thejns.org/doi/abs/10.3171/2017.4.SPINE16831

KEY WORDS alignment; cervical spondylotic myelopathy; laminoplasty; laminectomy; posterior spinal fusion

$\mathrm{D}$ EGENERATIVE cervical spondylosis can result in spinal cord compression and injury, which can lead to myelopathy. ${ }^{14}$ Cervical spondylotic myelopathy (CSM) often involves multiple vertebral levels, and evidence regarding the natural history of CSM suggests that $20 \%-60 \%$ of patients will deteriorate neurologically. ${ }^{8}$ CSM can be treated through an anterior and/or posterior approach to the spine, and both approaches have been proven to be effective for multilevel disease. ${ }^{12}$ The two posterior approaches traditionally used are laminoplasty,

ABBREVIATIONS CSM = cervical spondylotic myelopathy; EBL = estimated blood loss; JOA = Japanese Orthopaedic Association; LOS = length of stay; LPSF = laminectomy with posterior spinal fusion; SVA = sagittal vertical axis; VAS = visual analog scale.

SUBMITTED July 12, 2016. ACCEPTED April 10, 2017.

INCLUDE WHEN CITING Published online September 1, 2017; DOI: 10.3171/2017.4.SPINE16831. 
and laminectomy with posterior spinal fusion (LPSF). Laminectomy alone is generally avoided given the significant risk for delayed postlaminectomy kyphosis. ${ }^{2}$

The indications for using either laminoplasty or LPSF for multilevel CSM depend on a variety of demographic, social, and clinical factors. There are several clinical and radiological factors that are often taken into account when deciding between the two surgical techniques: presence of neck pain, degree of movement with flexion-extension, and cervical curvature. Generally, LPSF is preferred in patients with neck pain, significant translational movement, and/or cervical kyphosis. The rationale is that LPSF is able to stabilize the spinal column, thus offering superior pain relief compared with laminoplasty. The decision to use either procedure is based on each individual case and surgeon familiarity. ${ }^{5,13,27}$

Over the past 5-10 years, there has been a greater understanding of the relation of regional and global spinal alignment with functional and pain outcomes. In particular, cervical alignment has been suggested as an important factor associated with neck pain. ${ }^{9,15,20,23}$ We sought to assess whether LPSF allows for greater neck pain relief when compared with laminoplasty for multilevel CSM in cohorts of patients with similar postoperative cervical lordosis. This study compared perioperative and followup outcomes of patients with matched cervical sagittal alignment who had undergone either laminoplasty or LPSF. The analysis of neck pain outcomes takes into consideration the presence of preoperative pain via subgroup analysis. Additionally, we attempted to define the relationship between cervical sagittal curvatures and neck pain outcomes.

\section{Methods \\ Study Cohort}

This study was formally approved by the Committee of Human Research at the University of California, San Francisco. A consecutive cohort of patients diagnosed with multilevel CSM based on physical examination and radiographic imaging was identified from the time period January 2011 to December 2014. Patients who underwent posterior decompression of the cervical spine via laminoplasty or LPSF were identified and included in the study. Patients who underwent prior cervical spine surgery were excluded (the majority of whom were in the LPSF group). Matched cohorts of cervical lordosis were obtained by excluding patients with LPSF who had postoperative cervical sagittal Cobb angle measurements outside the range of the patients in the laminoplasty group. Patients underwent surgery by either 1 of 2 senior spine surgeons (P.V.M. and D.C). All laminoplasties were performed with the Hirabayashi technique (single-hinge door; Fig. 1) with plating and LPSF was performed with screw-rod fixation (Fig. 2). ${ }^{6}$ The selection of which surgical technique to use was considered on an individual case-by-case basis. The indications to proceed with either LPSF or laminoplasty for patients with multilevel CSM depended on clinical symptoms and radiological findings. LPSF was offered to patients who had significant neck pain and/or needed bilateral foraminotomies. Candidates for laminoplasties

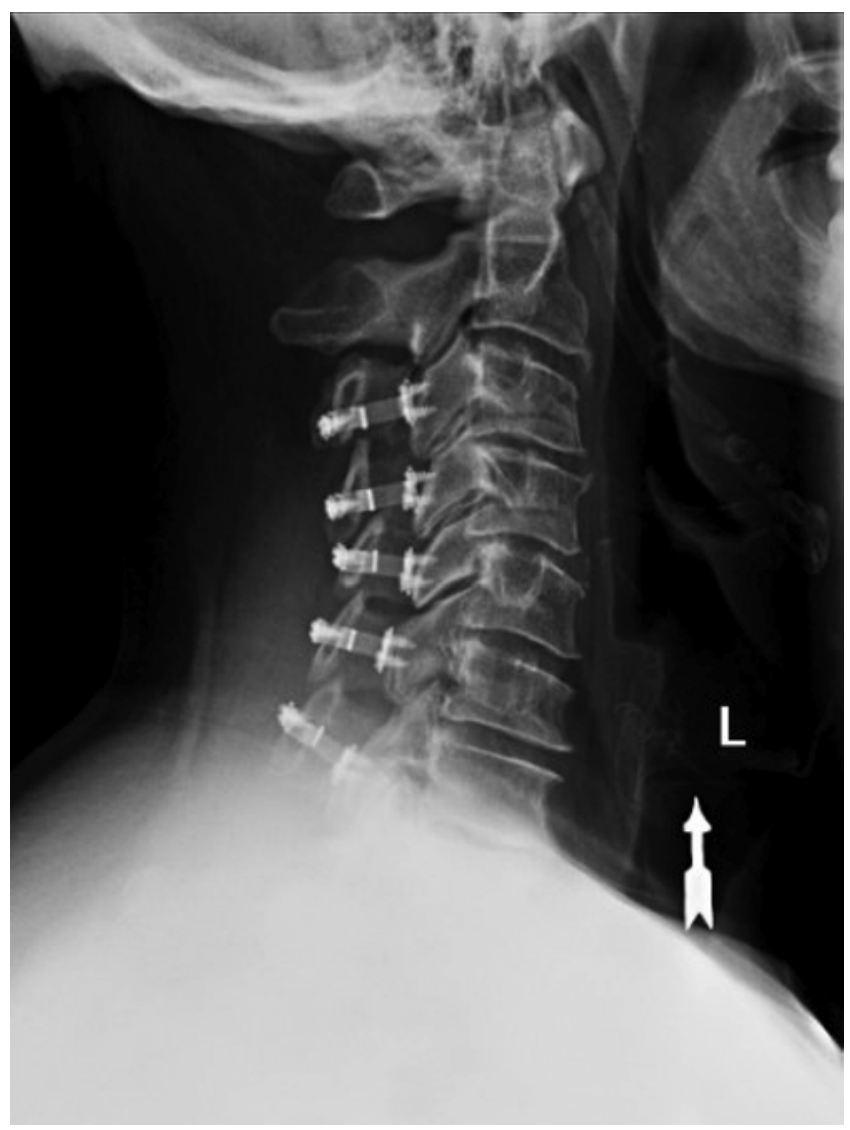

FIG. 1. Postoperative neutral lateral cervical radiograph showing C3-7 Hirabayashi (single-hinge door) laminoplasty.

tend to be patients who have a neutral or lordotic cervical spine, absence of listhesis (slip), and/or have a unilateral foraminal compression causing radiculopathy. In general, the decision to pursue either LPSF or laminoplasty was case based and patient wishes were taken into account if they fit indications for both procedures.

\section{Data Collection}

A comprehensive retrospective review of medical records was performed to gather data concerning patient demographics, clinical outcomes, and radiographic measures. Patient age and sex were recorded. Pain and neurological outcomes were reported based on the presence or absence of neck pain, visual analog scale (VAS) neck pain score, and Nurick myelopathy score. ${ }^{17}$ The VAS was measured from 0 to 10 , with 0 representing no pain and 10 representing the worst pain. The radiographic outcomes of interest were cervical lordosis, cervical sagittal vertical axis (SVA), and T-1 slope, which were all measured on neutral upright lateral cervical spine radiographs. Cervical lordosis was measured by the $\mathrm{C} 2-7 \mathrm{Cobb}$ angle (inferior endplate of C-2 to inferior endplate of C-7). Cervical SVA was measured as the distance between a plumb line from the C-2 centroid to a plumb line from the C-7 superior posterior endplate. The T-1 slope was defined as the angle between a horizontal line and the superior end plate of T-1. The methodology of these measurements can be 


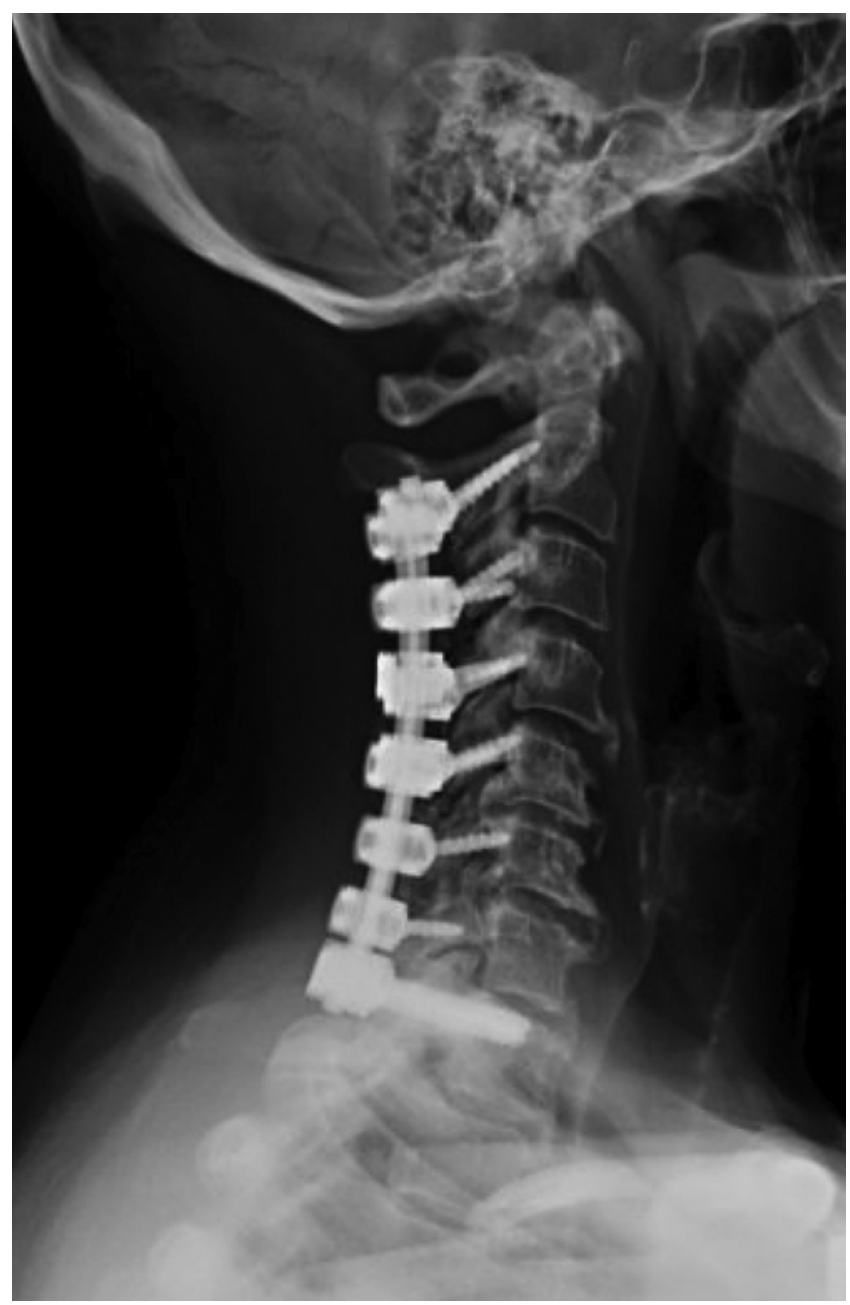

FIG. 2. Postoperative neutral lateral cervical radiograph showing C2-T1 LPSF.

observed in Fig. 3. All of these clinical and radiographic parameters were measured preoperatively and at the most recent follow-up. Perioperative outcomes that were collected included estimated blood loss (EBL; in milliliters), complications, and hospital length of stay (LOS; date of surgery to date of discharge). Complications were defined as any unforeseen event requiring additional medical and/ or surgical intervention. Readmissions within 30 days of index surgical hospitalization were recorded. Patient longterm complications (events that occurred after discharge) and reoperations were evaluated.

\section{Statistical Analysis}

The cohort was stratified into two groups: patients who underwent laminoplasty and patients who underwent LPSF. Demographics, baseline clinical variables, and outcome measures were compared between the two groups. Follow-up pain outcomes underwent a subgroup analysis to control for preoperative neck pain; for this analysis, the cohort was first separated into two groups: patients with preoperative pain and patients without preoperative pain. Then, among those groups, pain outcomes were compared between patients who underwent laminoplasty and those who underwent LPSF. An additional analysis was performed to determine the relationship between degree of cervical lordosis and neck pain outcomes. For this analysis, patients were categorized into 5 groups based on postoperative cervical lordosis $\left(<5^{\circ}, 5^{\circ}-10^{\circ}, 11^{\circ}-15^{\circ}, 16^{\circ}-20^{\circ}\right.$, and $>20^{\circ}$ ). Pain outcomes were then compared in the total cohort, in laminoplasty patients, and in LPSF patients. A bivariate analysis was then performed to identify a cervical lordosis threshold. Bivariate analyses were performed using either a chi-square test for categorical outcomes or a 2-tailed Student t-test for continuous outcomes. A p value $<0.050$ was the threshold for statistical significance. All statistical analyses were performed using SAS 9.4 (SAS, Inc.).

\section{Results}

In the process of generating the matched cohort based on postoperative cervical sagittal Cobb angles, 8 LPSF patients were excluded based on postoperative cervical sagittal Cobb angle. This resulted in postoperative cervical sagittal Cobb angles that ranged from $-6^{\circ}$ (kyphosis) to $45^{\circ}$ (lordosis) for laminoplasty patients and $-4^{\circ}$ (kyphosis) to $35^{\circ}$ (lordosis) for LPSF patients. A total of 145 patients were included in the final analysis. There were 101 laminoplasty patients and 44 patients who underwent LPSF. The mean age of all patients was 63.0 years, and $65.5 \%$ were men (Table 1). Significantly more men underwent laminoplasty than LPSF (73.3\% vs. 47.7\%, p = 0.004). Table 1 also shows the comparisons of preoperative pain and neurological baselines. The overall incidence of preoperative neck pain was $66.2 \%$ and the mean VAS neck pain score was 6.1 (Table 1). In regard to preoperative pain, patients who underwent LPSF had a higher incidence of pain (laminoplasty $62.4 \%$ vs LPSF $75.0 \%, \mathrm{p}=0.182$ ) and mean VAS neck pain score (laminoplasty 5.6 vs LPSF 6.9, $\mathrm{p}=0.051$ ), but these differences were not statistically significant. The overall mean Nurick scale score was 2.1 and both groups had identical scores (2.1 vs 2.1, p = 0.836). The mean preoperative cervical sagittal Cobb angle for the entire cohort was $9.1^{\circ}$ of lordosis (Table 1). Patients who underwent LPSF had significantly less preoperative cervical lordosis compared with patients who underwent laminoplasty $\left(5.8^{\circ}\right.$ vs $10.9^{\circ}$, respectively; $\mathrm{p}=0.018$ ). The mean preoperative cervical SVA and T-1 slopes were $28.0 \mathrm{~mm}$ and $24.8^{\circ}$, respectively. Between the two groups, there were no significant differences in preoperative cervical SVA (27.4 vs 28.7 $\mathrm{mm}, \mathrm{p}=0.761)$ or $\mathrm{T}-1$ slope $\left(25.7^{\circ}\right.$ vs. $\left.23.8^{\circ}, \mathrm{p}=0.310\right)$.

Table 2 compares perioperative outcomes between patients who underwent laminoplasty and those who underwent LPSF. The mean number of levels operated as an entire cohort was 4.6 levels. There was a statistically significant difference in the number of levels operated between the two groups, but the actual difference was small: 4.3 levels for laminoplasty and 5.3 for LPSF ( $p<0.001)$. Mean EBL was $236.3 \mathrm{ml}$. LPSF was associated with significantly greater blood loss compared with laminoplasty (325.0 ml vs $196.6 \mathrm{ml}$, respectively; $\mathrm{p}<0.001)$. The overall perioperative surgical complication rate was $8.3 \%$, and the mean hospital LOS was 3.7 days. There was no significant difference between the two groups for perioperative 

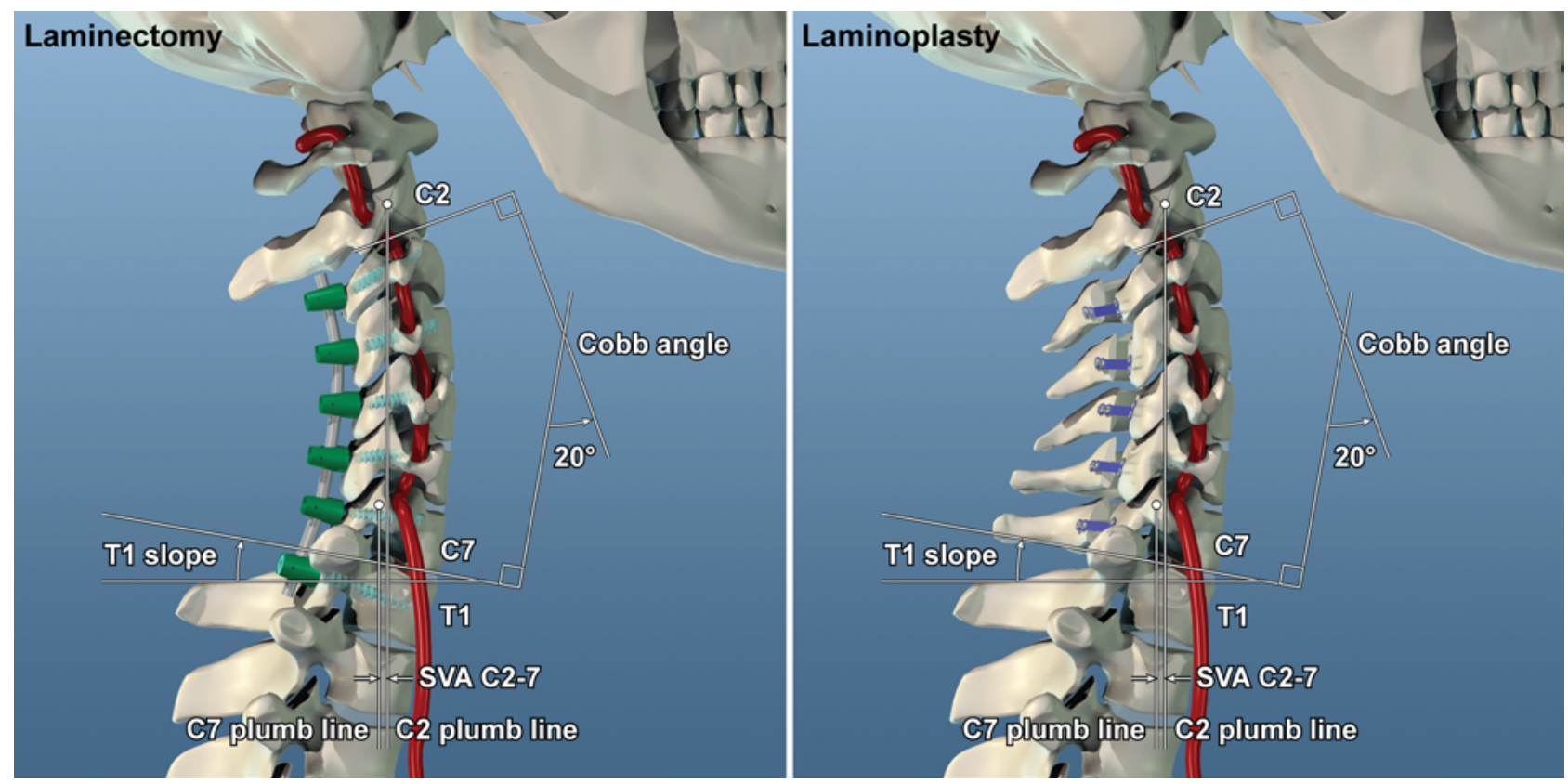

FIG. 3. Artist illustration of LPSF (left) and laminoplasty (right) with cervical sagittal Cobb angles of $20^{\circ}$ of lordosis. C2-7 SVA and T-1 slope measurements are also shown. Copyright Darryl Lau. Published with permission.

complication rate (laminoplasty $6.9 \%$ vs LPSF $11.4 \%, \mathrm{p}=$ 0.512 ) or hospital LOS (laminoplasty 3.5 days vs LPSF 4.3 days, $\mathrm{p}=0.054)$. The overall 30 -day readmission rate was $3.4 \%$, and there was no significant difference between the groups for this variable $(3.0 \%$ vs $4.5 \%, \mathrm{p}=0.639)$.

Table 3 compares long-term complication rates, followup neurological status, and follow-up cervical Cobb angle in the two groups. Of 134 patients, $92.4 \%$ were available for follow-up and the mean follow-up length was 17.3 months. Both groups had similar mean follow-up durations (laminoplasty 17.4 months vs LPSF 16.8 months, $\mathrm{p}$ $=0.754)$. The overall long-term complication and reoperation rates were both $5.2 \%$. Patients who underwent LPSF had significantly higher long-term complication rates than laminoplasty patients $(11.6 \%$ vs $2.2 \%, \mathrm{p}=0.036)$. However, reoperation rates were similar between both groups $(4.4 \%$ vs $7.0 \%, p=0.681)$. The reasons for reoperation in the laminoplasty group were fracture at an adjacent level following a traumatic fall $(\mathrm{n}=1)$, adjacent-segment stenosis resulting in myelopathy $(\mathrm{n}=1)$, and wound infections ( $\mathrm{n}$ $=2$ ). The reasons for reoperation in the LPSF group were pseudarthrosis $(n=1)$ and wound infections $(n=2)$. The mean follow-up Nurick scale score improved from a preoperative score of 2.1 to a follow-up score of 1.2. Patients who underwent LPSF had a significantly lower (improved) Nurick score (0.9) compared with patients who underwent laminoplasty $(1.4, \mathrm{p}=0.014)$. The overall mean postoperative cervical Cobb angle was $8.2^{\circ}$ of lordosis. Because this study was established to have matched cohorts of cervical lordosis, there was no significant difference in postoperative cervical Cobb angles between the two groups $\left(8.8^{\circ}\right.$ vs $7.1^{\circ}$ of lordosis, $\left.\mathrm{p}=0.454\right)$. Mean follow-up cervical SVA and T-1 slope were $31.1 \mathrm{~mm}$ and $26.4^{\circ}$, respectively. There were no significant differences in cervical SVA (29.8 vs $32.5 \mathrm{~mm}, \mathrm{p}=0.501)$ or $\mathrm{T}-1$ slope $\left(26.2^{\circ}\right.$ vs $\left.26.5^{\circ}, \mathrm{p}=0.927\right)$ between groups.

TABLE 1. Comparison of demographics and preoperative clinical baselines in patients who underwent laminoplasty versus LPSF

\begin{tabular}{lcccc}
\hline \multicolumn{1}{c}{ Variable } & Total & Laminoplasty & LPSF & p Value \\
\hline No. of patients & 145 & 101 & 64 & $0.9 \pm 9.0$ \\
\hline Age (yrs) & $63.0 \pm 11.2$ & $63.9 \pm 11.9$ & $21(47.7)$ & 0.142 \\
\hline Males (\%) & $95(65.5)$ & $74(73.3)$ & $33(75.0)$ & 0.004 \\
\hline Preop neck pain (\%) & $96(66.2)$ & $63(62.4)$ & $6.9 \pm 2.4$ & 0.182 \\
\hline Preop neck VAS score & $6.1 \pm 2.6$ & $5.6 \pm 2.6$ & $2.1 \pm 1.4$ & 0.051 \\
\hline Preop Nurick scale score & $2.1 \pm 1.3$ & $2.1 \pm 1.3$ & $5.8 \pm 12.3$ & 0.836 \\
\hline Preop cervical Cobb angle $\left(^{\circ}\right)$ & $9.1 \pm 11.4$ & $10.9 \pm 10.4$ & $28.7 \pm 13.1$ & 0.018 \\
\hline Preop cervical SVA (mm) & $28.0 \pm 12.7$ & $27.4 \pm 12.0$ & $23.8 \pm 6.1$ & 0.761 \\
\hline Preop T-1 slope $\left(^{\circ}\right)$ & $24.8 \pm 5.7$ & $25.7 \pm 5.0$ & 0.310 \\
\hline
\end{tabular}

Data given as mean \pm SD unless otherwise indicated. 
TABLE 2. Comparison of perioperative outcomes between patients who underwent laminoplasty versus LPSF

\begin{tabular}{lcccc}
\hline \multicolumn{1}{c}{ Periop Outcome } & Total & Laminoplasty & LPSF & p Value \\
\hline Mean no. of levels operated \pm SD & $4.6 \pm 1.2$ & $4.3 \pm 0.9$ & $5.3 \pm 1.7$ & $<0.001$ \\
\hline Mean EBL \pm SD (ml) & $236.3 \pm 209.3$ & $196.6 \pm 203.2$ & $325.0 \pm 192.4$ & $<0.001$ \\
\hline Periop complications (\%) & $12(8.3)$ & $7(6.9)$ & $5(11.4)$ & 0.512 \\
\hline Mean LOS \pm SD (days) & $3.7 \pm 2.3$ & $3.5 \pm 2.5$ & $4.3 \pm 1.8$ & 0.054 \\
\hline No. of 30-day readmissions (\%) & $5(3.4)$ & $3(3.0)$ & $2(4.5)$ & 0.639 \\
\hline
\end{tabular}

Follow-up pain incidence and VAS neck pain scores are listed in Table 4. Overall, the incidence of neck pain at follow-up was $29.1 \%$, and there was no significant difference in pain incidence between patients who underwent laminoplasty $(29.7 \%)$ versus LPSF $(27.9 \%, \mathrm{p}=0.834)$. The mean follow-up VAS neck pain score was 1.6. There was no significant difference in VAS neck pain scores between the two groups as well (1.6 vs $1.7, \mathrm{p}=0.996)$. To further normalize the analysis of pain outcomes, comparisons were made among subgroups in patients with and without preoperative neck pain. In patients with preoperative neck pain, 34.5\% had pain at follow-up and the mean VAS neck pain score was 2.0. Among this subgroup, there were no significant differences in follow-up pain incidence $(36.4 \%$ vs $31.3 \%, p=0.629)$ and VAS neck pain scores (2.1 vs $1.8, \mathrm{p}=0.731$ ). In patients without preoperative neck pain, $19.2 \%$ had pain at follow-up and the mean VAS score was 1.0. Similarly, there was no significant difference in follow-up pain incidence $(19.4 \%$ vs $18.2 \%, \mathrm{p}=0.926)$ and VAS neck pain scores $(1.0$ vs $1.1, \mathrm{p}=0.908)$.

Tables 5 and 6 demonstrate the relationships between postoperative cervical sagittal Cobb angle and neck pain incidence and severity. As noted in Table 5, there was a significant association between pain outcomes with cervical curvature; as cervical lordosis increased, there was a decrease in the incidence $(p=0.017)$ and severity of neck pain ( $\mathrm{p}=0.008$; Figs. 4 and 5). At increments of $5^{\circ}$ (from $<5^{\circ}$ to $>20^{\circ}$ of cervical lordosis), follow-up pain incidence decreased from $44.9 \%$ to $5.6 \%$, and the VAS neck pain score decreased from 2.7 to 0.4 . This association was also observed when laminoplasty patients were isolated for analysis. Pain incidence significantly decreased from $50.0 \%$ to $0.0 \%(\mathrm{p}=0.010)$ and VAS neck pain scores decreased from 2.9 to $0.0(\mathrm{p}=0.004)$. A case example can be seen in Fig. 6 in which a patient with cervical kyphosis underwent multilevel laminoplasty and had ongoing severe neck pain at the 2-year follow-up. When more than $20^{\circ}$ of postoperative lordosis was present, laminoplasty patients had a significant and profound decrease in pain incidence (34.6\% vs $0.0 \%, \mathrm{p}=0.011)$ and VAS scores $(1.9$ vs $0.0, p=0.018$ ) compared with patients with less lordosis (Table 6). Figure 3 shows an illustration demonstrating the importance of this particular finding. This trend was not as substantial in LPSF patients. Among patients with postoperative cervical Cobb measurements greater than $20^{\circ}$ of lordosis, there was no significant difference in mean preoperative cervical sagittal Cobb angles between LPSF and laminoplasty patients $\left(17.4^{\circ}\right.$ vs $21.8^{\circ}$, respectively; $p$ $=0.499$ ).

A post hoc analysis concentrating on laminoplasty patients was performed to determine whether there were preoperative factors that would indicate which patients would be more likely to achieve greater than $20^{\circ}$ of cervical lordosis after surgery. The only preoperative parameter that was significantly associated with postoperative cervical lordosis of more than $20^{\circ}$ was preoperative cervical lordosis. We found that patients with postoperative cervical lordosis greater than $20^{\circ}$ had a mean preoperative cervical lordosis that was significantly greater than patients with $20^{\circ}$ or less of cervical lordosis $\left(21.8^{\circ}\right.$ vs $9.0^{\circ}$, $\mathrm{p}<0.001)$.

\section{Discussion}

The laminoplasty procedure was originally developed for the treatment of stenosis of the cervical canal due to ossification of the posterior longitudinal ligament, and since then this procedure has been used to treat various

TABLE 3. Comparison of follow-up outcomes between patients who underwent laminoplasty versus LPSF

\begin{tabular}{lcccc}
\hline \multicolumn{1}{c}{ Variable } & Total & Laminoplasty & LPSF & p Value \\
\hline No. of patients & 134 & 91 & 43 & \\
\hline Long-term complications (\%) & $7(5.2)$ & $2(2.2)$ & $5(11.6)$ & 0.036 \\
\hline Reop (\%) & $7(5.2)$ & $4(4.4)$ & $3(7.0)$ & 0.681 \\
\hline Follow-up Nurick scale score & $1.2 \pm 1.4$ & $1.4 \pm 1.5$ & $0.9 \pm 1.3$ & 0.014 \\
\hline Follow-up cervical Cobb angle $\left(^{\circ}\right)$ & $8.2 \pm 11.4$ & $8.8 \pm 11.7$ & $7.1 \pm 11.0$ & 0.454 \\
\hline Follow-up cervical SVA (mm) & $31.1 \pm 11.8$ & $29.8 \pm 11.5$ & $32.5 \pm 11.5$ & 0.501 \\
\hline Follow-up T-1 slope $\left({ }^{\circ}\right)$ & $26.4 \pm 10.4$ & $26.2 \pm 5.4$ & $26.5 \pm 13.8$ & 0.927 \\
\hline Length of follow-up (mos) & $17.3 \pm 11.3$ & $17.4 \pm 12.3$ & $16.8 \pm 8.4$ & 0.754 \\
\hline
\end{tabular}

Data given as mean \pm SD unless otherwise indicated. 
TABLE 4. Patient comparison and subgroup analysis of follow-up pain outcomes for laminoplasty versus LPSF

\begin{tabular}{|c|c|c|c|c|}
\hline Variable & Total & Laminoplasty & LPSF & p Value \\
\hline \multicolumn{5}{|l|}{ Overall } \\
\hline No. of patients & 134 & 91 & 43 & \\
\hline Follow-up pain (\%) & $39(29.1)$ & $27(29.7)$ & $12(27.9)$ & 0.834 \\
\hline Mean follow-up VAS score \pm SD & $1.6 \pm 2.8$ & $1.6 \pm 2.7$ & $1.7 \pm 2.9$ & 0.996 \\
\hline \multicolumn{5}{|l|}{ Patients w/ preop pain } \\
\hline No. of patients & 87 & 55 & 32 & \\
\hline Follow-up pain (\%) & $30(34.5)$ & $20(36.4)$ & $10(31.3)$ & 0.629 \\
\hline Mean follow-up VAS score \pm SD & $2.0 \pm 3.0$ & $2.1 \pm 3.0$ & $1.8 \pm 3.0$ & 0.731 \\
\hline Mean follow-up cervical Cobb angle $\pm \operatorname{SD}\left({ }^{\circ}\right)$ & $7.6 \pm 10.1$ & $7.3 \pm 10.8$ & $8.1 \pm 9.0$ & 0.740 \\
\hline \multicolumn{5}{|l|}{ Patients w/o preop pain } \\
\hline No. of patients & 47 & 36 & 11 & \\
\hline Follow-up pain (\%) & $9(19.2)$ & $7(19.4)$ & $2(18.2)$ & 0.926 \\
\hline Mean follow-up VAS score \pm SD & $1.0 \pm 2.3$ & $1.0 \pm 2.2$ & $1.1 \pm 2.5$ & 0.908 \\
\hline Mean follow-up cervical Cobb angle $\pm \operatorname{SD}\left({ }^{\circ}\right)$ & $9.4 \pm 12.8$ & $10.9 \pm 12.8$ & $4.5 \pm 15.5$ & 0.170 \\
\hline
\end{tabular}

pathologies causing CSM. ${ }^{6,19}$ Several studies have demonstrated that laminoplasty offers neurological improvement (modified Japanese Orthopaedic Association [JOA], Odom, and Nurick scales) similar to that of LPSF in the treatment of multilevel CSM. 5,25,27 The decision as to which surgical procedure to perform is not always transparent and often depends on a multitude of patient- and clinically related factors, such as age, medical morbidity (risk for surgery), neck pain, cervical lordosis, axial movement with neck flexion-extension, and social factors. Traditionally, LPSF is offered to patients with concomitant neck pain in addition to CSM. The concept is that internal fixation minimizes movement, which helps alleviate musculoskeletal pain. Over the past decade, there has been an accumulation of evidence suggesting that pain outcomes are strongly related to regional and global spinal alignment. ${ }^{3,18,21}$ More specific findings have also been shown to be true in relation to neck pain and cervical spinal align- ment. However, the effect of cervical sagittal alignment on neck pain has not been thoroughly studied in patients who undergo laminoplasty or LPSF. ${ }^{23}$

This study compared perioperative and follow-up outcomes of matched cohorts of patients with postoperative cervical lordosis who underwent either laminoplasty or LPSF. Additionally, the patients had similar follow-up cervical SVAs and T-1 slopes. The findings presented suggest that LPSF is associated with slightly greater blood loss, longer hospital stays, and higher long-term complication rates than laminoplasty. However, greater neurological recovery was observed in patients who underwent LPSF. In regard to neck pain outcomes, there was no difference in incidence and VAS neck pain scores at follow-up in patients who presented either with or without preoperative neck pain after undergoing laminoplasty or LPSF. However, in patients who underwent laminoplasty, greater cervical lordosis was significantly associated with better pain

TABLE 5. Distribution of pain incidence and severity based on cervical sagittal Cobb angle

\begin{tabular}{|c|c|c|c|c|c|c|}
\hline \multirow[b]{2}{*}{ Variable } & \multicolumn{5}{|c|}{ Cervical Sagittal Cobb Angle $\left({ }^{\circ}\right)$} & \multirow[b]{2}{*}{ p Value } \\
\hline & $<5$ & $5-10$ & $11-15$ & $16-20$ & $>20$ & \\
\hline \multicolumn{7}{|l|}{ Entire cohort } \\
\hline No. of patients & 49 & 34 & 21 & 12 & 18 & \\
\hline Neck pain incidence (\%) & $22(44.9)$ & $9(26.5)$ & $4(19.1)$ & $3(25.0)$ & $1(5.6)$ & 0.017 \\
\hline Mean VAS neck pain score \pm SD & $2.7 \pm 3.4$ & $1.9 \pm 2.3$ & $1.0 \pm 2.1$ & $1.1 \pm 2.2$ & $0.4 \pm 1.9$ & 0.008 \\
\hline \multicolumn{7}{|l|}{ Laminoplasty } \\
\hline No. of patients & 34 & 24 & 9 & 11 & 13 & \\
\hline Neck pain incidence (\%) & $17(50.0)$ & $5(20.8)$ & $2(22.2)$ & $3(27.3)$ & $0(0.0)$ & 0.010 \\
\hline Mean VAS neck pain score \pm SD & $2.9 \pm 3.3$ & $1.0 \pm 2.1$ & $1.2 \pm 2.4$ & $1.2 \pm 2.2$ & $0.0 \pm 0.0$ & 0.004 \\
\hline \multicolumn{7}{|l|}{ LPSF } \\
\hline No. of patients & 15 & 10 & 12 & 1 & 5 & \\
\hline Neck pain incidence (\%) & $5(33.3)$ & $4(40.0)$ & $2(16.7)$ & $0(0.0)$ & $1(20.0)$ & 0.691 \\
\hline Mean VAS neck pain score \pm SD & $2.7 \pm 3.5$ & $1.9 \pm 2.8$ & $0.8 \pm 1.9$ & $0.0 \pm N A$ & $1.6 \pm 3.6$ & 0.691 \\
\hline
\end{tabular}

$\mathrm{NA}=$ not applicable. 
TABLE 6. Distribution of pain incidence and severity based on cervical sagittal Cobb angle $\leq$ or $>20^{\circ}$

\begin{tabular}{|c|c|c|c|}
\hline \multirow[b]{2}{*}{ Variable } & \multicolumn{2}{|c|}{$\begin{array}{l}\text { Cervical Sagittal } \\
\text { Cobb Angle }\left({ }^{\circ}\right)\end{array}$} & \multirow{2}{*}{$\begin{array}{c}p \\
\text { Value }\end{array}$} \\
\hline & $\leq 20$ & $>20$ & \\
\hline \multicolumn{4}{|l|}{ Entire cohort } \\
\hline No. of patients & 116 & 18 & \\
\hline Neck pain incidence (\%) & $38(32.8)$ & $1(5.6)$ & 0.018 \\
\hline Mean VAS neck pain score \pm SD & $1.8 \pm 2.8$ & $0.4 \pm 1.9$ & 0.047 \\
\hline \multicolumn{4}{|l|}{ Laminoplasty } \\
\hline No. of patients & 78 & 13 & \\
\hline Neck pain incidence (\%) & $27(34.6)$ & $0(0.0)$ & 0.011 \\
\hline Mean VAS neck pain score \pm SD & $1.9 \pm 2.9$ & $0.0 \pm 0.0$ & 0.018 \\
\hline \multicolumn{4}{|l|}{ LPSF } \\
\hline No. of patients & 38 & 5 & \\
\hline Neck pain incidence (\%) & $11(29.0)$ & $1(20.0)$ & 0.675 \\
\hline Mean VAS neck pain score \pm SD & $1.7 \pm 2.8$ & $1.6 \pm 3.6$ & 0.967 \\
\hline
\end{tabular}

outcomes, especially when more than $20^{\circ}$ of lordosis was present.

Cervical LPSF is generally accepted as a more invasive surgery with higher morbidity than laminoplasty. This is not surprising as LPSF requires additional muscle exposure (dissection to the lateral masses), spinal fixation, and bone arthrodesis, which can result in greater intraoperative blood loss and postoperative pain. ${ }^{4,24}$ Furthermore, pseudarthrosis becomes a concern with LPSF over time, and reported rates are as high as $38 \% .{ }^{27}$ However, the actual difference in perioperative morbidity and complications between multilevel laminoplasty and LPSF is relatively small and patients tolerate both procedures well. In fact, it has been suggested that when addressing more than 4 levels, postoperative pain following laminoplasty is greater than that in LPSF. ${ }^{16}$ In regard to blood loss, our study showed a mean difference of $128.4 \mathrm{ml}$ between the two groups; this difference is likely not clinically significant in adults. For the treatment of multilevel CSM, reported perioperative complication rates following laminoplasty range between $6 \%$ and $13 \%, 1,24,25$ and for LPSF between $9 \%$ and $15 \% .1,24,25$ These rates are similar to what was observed in our study. The difference in complication rates may be less substantial than previously believed. We did, however, observe higher rates of long-term complications with LPSF; 3 of the 5 complications were pseudarthrosis, a risk not found with laminoplasty. Nonetheless, reoperations rates were similar between the two groups and this is consistent with observations in a study by Adogwa et al., who reported reoperation rates of $10 \%$ in LPSF and $7 \%$ in laminoplasty patients. ${ }^{1}$

Interestingly, in our cohort, patients who underwent LPSF were observed to have greater neurological improvement than patients who underwent laminoplasty at followup. The mean postoperative Nurick score for patients who underwent LPSF was 0.9 (no signs of myelopathy) and the mean Nurick score for laminoplasty patients was 1.4 (ongoing signs of myelopathy). This finding is different from what is reported from past studies. Lee et al. and Yoon et al. individually performed systematic reviews comparing

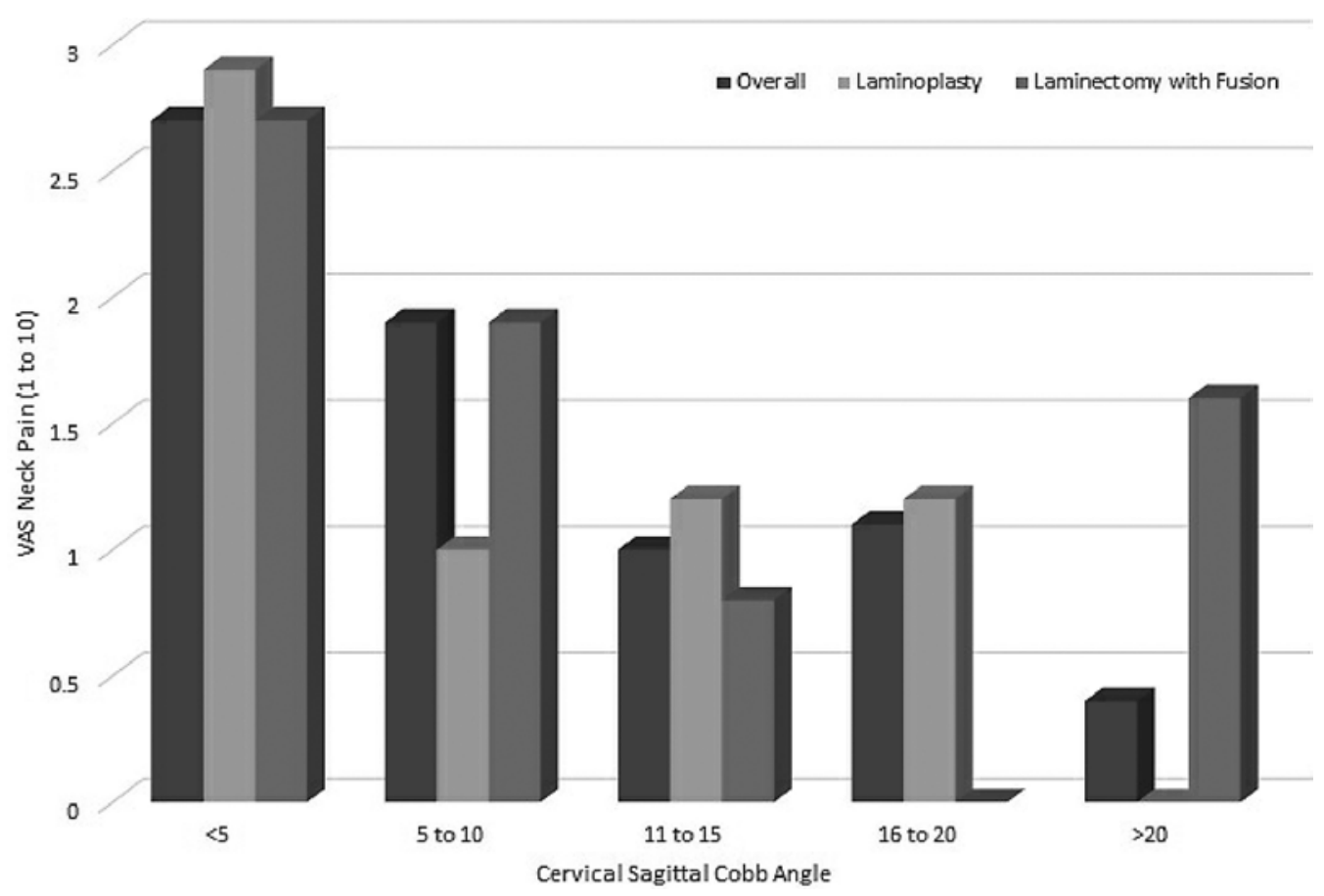

FIG. 4. Graph showing the relationship between neck pain severity and degree of cervical lordosis following either laminoplasty or LPSF. As an entire cohort, there was a significant association between neck pain VAS scores and cervical sagittal Cobb angle. Greater cervical lordosis correlated with lower neck pain VAS scores $(p=0.008)$. Similarly, this same trend was noted in laminoplasty patients $(p=0.004)$, but not in patients who underwent LPSF $(p=0.691)$. 


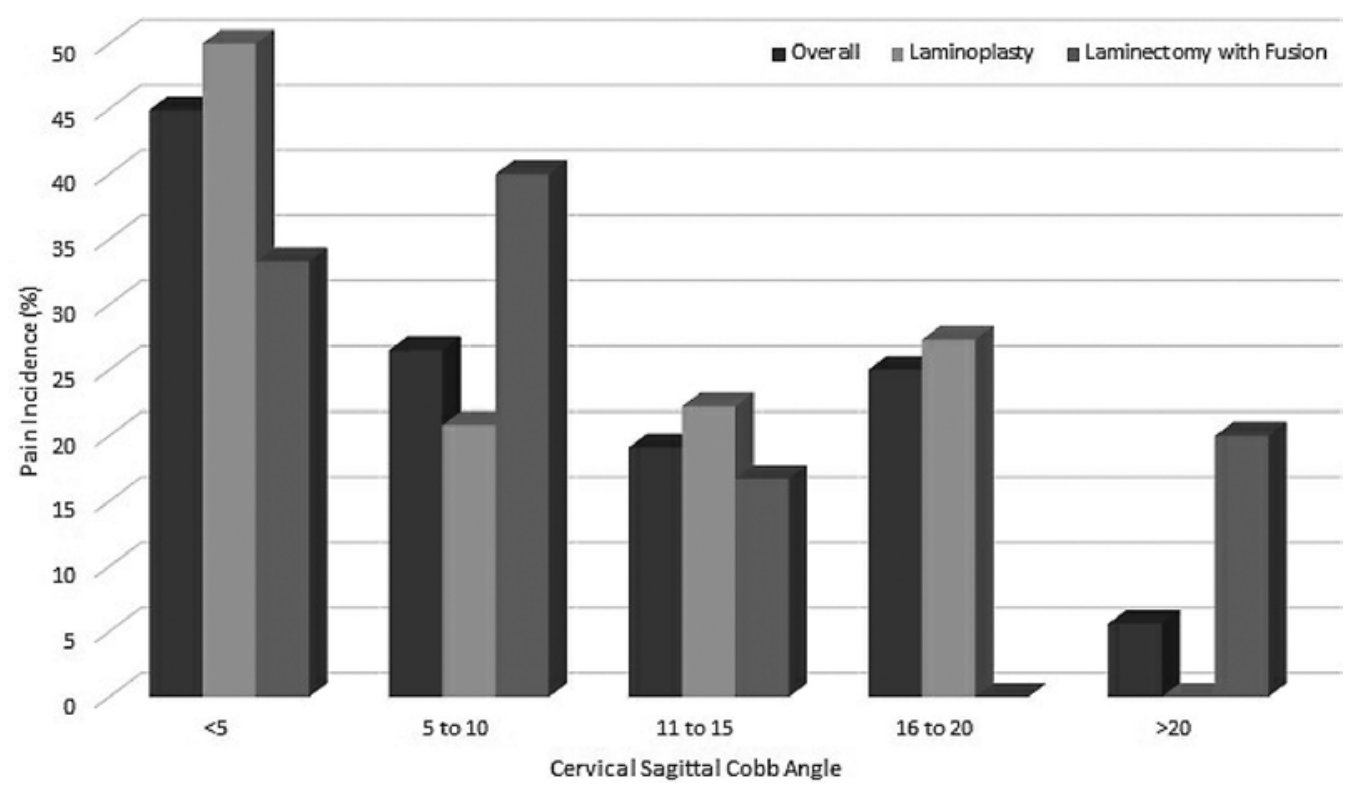

FIG. 5. Graph showing the relationship between neck pain incidence and degree of cervical lordosis following either laminoplasty or LPSF. As an entire cohort, there was a significant association between neck pain incidence and cervical sagittal Cobb angle. Greater cervical lordosis correlated with lower neck pain incidence $(p=0.017)$. Similarly, this same trend was noted in laminoplasty patients $(p=0.010)$, but not in patients who underwent LPSF $(p=0.691)$.

neurological outcomes in patients who underwent laminoplasty or laminectomy for CSM, and they did not find a significant difference in follow-up JOA scale scores..$^{10,27}$ Similarly, Highsmith et al. showed similar magnitudes of modified JOA scale score improvement at follow-up: 2.7 points in laminoplasty patients and 2.8 points in LPSF patients. ${ }^{5}$ It is unclear why a discrepancy exists between our findings and those of past studies, but a potential reason for the variance could be the different modalities used to measure neurological outcome and cervical myelopathy. Nurick score is mainly a measure of lower extremity function while other scales such as the modified JOA scale measure myelopathy in greater detail. Another major difference between past studies and our current study is our use of cohorts with matched cervical alignment. Intrinsic closure of the lamina resulting in less decompression following laminoplasty is a genuine concern, and there is evidence that cervical alignment has an effect on the degree of laminar closure following laminoplasty. ${ }^{26}$ The reported incidence of laminar closure ranges from $39 \%$ to $79 \%$ depending on the laminoplasty technique used. ${ }^{26}$ Laminar closure can result in a $10 \%$ decrease in diameter size of the decompressed spinal canal. ${ }^{11}$ When laminar closure occurs, this process can result in $30 \%$ less central canal decompression compared with laminectomy. ${ }^{22}$ Laminectomy intrinsically may also offer greater decompression because after removing the lamina the surgeons have direct access to removing compressive ligaments (not readily accessed during laminoplasty) and even extending decompressions laterally if warranted. In patients with severe canal stenosis, this extra decompression may make a clinical difference, potentially offering a greater chance of neurological recovery.

Neck pain is a challenging entity to treat. Understanding the underlying cause of pain is key in implementing the ideal management plan. Over the past 10 years, we have learned a great deal about how regional and global spinal alignment influences pain and functional outcomes. ${ }^{9,20}$ It has been shown that cervical lordosis and C2-7 SVA are correlated with clinical measures of regional disability (improved cervical alignment correlated positively with healthrelated quality of life outcomes). In patients with multilevel CSM, neck pain is typically an indication to offer LPSF because previous studies have demonstrated a high incidence of new neck pain following laminoplasty, as high as $25 \%$ of patients. ${ }^{7,27}$ However, according to the findings from this study among patients with matched postoperative cervical alignment and lordosis, follow-up pain incidence and severity were similar for patients who underwent laminoplasty or LPSF, despite the presence or absence of preoperative neck pain. This may suggest that postoperative cervical alignment and curve is a significant determinant in influencing the efficacy of either laminoplasty or LPSF in treating neck pain. According to our data, the degree of cervical lordosis is particularly relevant to patients who undergo multilevel laminoplasty. Half of the laminoplasty patients with follow-up cervical lordosis of less than $5^{\circ}$ continued to experience ongoing neck pain. But as cervical lordosis increased toward the normal range there was a clear association with better neck pain outcomes. Based on a systematic review by Kuntz et al., there is a great variability in degrees of normal cervical lordosis in asymptomatic neutral upright adults with $\mathrm{C} 2-7 \mathrm{Cobb}$ angles ranging from $13^{\circ}$ to $21^{\circ}$ and a mean $17^{\circ}$ of lordosis. ${ }^{9}$ Based on the data from this study, it appears that more than $20^{\circ}$ of cervical lordosis may be the most ideal in offering the best neck pain outcomes. It seems that the presence of cervical lordosis before surgery is a factor associated with being able to achieve lordosis greater than $20^{\circ}$ after laminoplasty. This threshold needs to be evaluated in a larger patient cohort to translate these findings directly into clinical practice. 


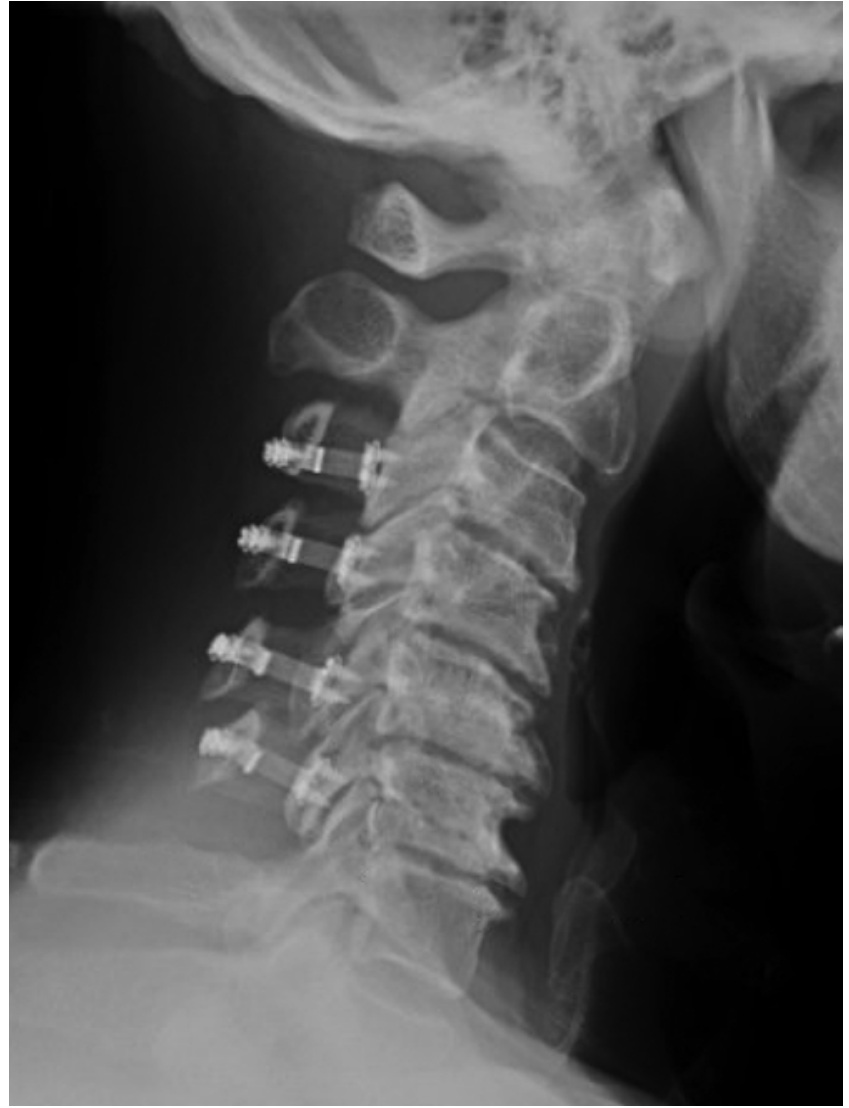

FIG. 6. Postoperative neutral lateral cervical radiograph showing C3-6 laminoplasty in a patient with cervical kyphosis. This is a case example in which a patient underwent multilevel laminoplasty and continued to have persistent neck pain at the 2-year follow-up. The sagittal cervical C2-7 Cobb angle was approximately $5^{\circ}$ of kyphosis.

The limitations of this study are generally related to the retrospective design. There is an inherent patient selection bias when comparing surgical procedures because indications for laminoplasty and LPSF tend to be different. Each patient is evaluated on a case-by-case basis and patient desires are taken into account in the decision-making process. Additionally, as in all retrospective reviews, there are various unknown confounders that cannot be assessed or accounted for that may affect the outcomes of interest. For this study, there is a small risk for selection bias in the process of generating a matched cohort by excluding LPSF patients based on postoperative cervical Cobb angle measurements. By excluding the LPSF outliers, some of which are kyphotic, there may be a bias in the results to support LPSF over laminoplasty. However, this bias is minimized and accounted for because our study includes subgroup analyses stratifying outcomes based on cervical lordosis. Additionally, the cervical SVA and T-1 slope were comparable in both groups before surgery and at follow-up. Future prospective studies or a randomized trial are needed to address these drawbacks.

\section{Conclusions}

Multilevel laminoplasty and LPSF are both safe and ef- fective procedures associated with low morbidity for the treatment of CSM. LPSF is associated with slightly greater blood loss and a higher long-term complication rate than laminoplasty, but the difference in magnitude may be less substantial than previously perceived. However, the findings from this study show that patients who had undergone LPSF had better neurological outcomes (resolution of myelopathy) compared with patients who underwent laminoplasty. In patients with similar postoperative cervical lordosis and sagittal alignment, the incidence and severity of neck pain are similar for patients undergoing either laminoplasty or LPSF. This finding highlights the importance of taking into account cervical lordosis during surgical planning and pursuing relatively normal cervical lordosis. Additionally, in patients who undergo laminoplasty, greater cervical lordosis may be associated with better neck pain outcomes, especially in patients with greater than $20^{\circ}$ of lordosis postoperatively.

\section{References}

1. Adogwa O, Huang K, Hazzard M, Chagoya G, Owens R, Cheng J, et al: Outcomes after cervical laminectomy with instrumented fusion versus expansile laminoplasty: a propensity matched study of 3185 patients. J Clin Neurosci 22:549-553, 2015

2. Albert TJ, Vacarro A: Postlaminectomy kyphosis. Spine (Phila Pa 1976) 23:2738-2745, 1998

3. Harroud A, Labelle H, Joncas J, Mac-Thiong JM: Global sagittal alignment and health-related quality of life in lumbosacral spondylolisthesis. Eur Spine J 22:849-856, 2013

4. Heller JG, Edwards CC II, Murakami H, Rodts GE: Laminoplasty versus laminectomy and fusion for multilevel cervical myelopathy: an independent matched cohort analysis. Spine (Phila Pa 1976) 26:1330-1336, 2001

5. Highsmith JM, Dhall SS, Haid RW Jr, Rodts GE Jr, Mummaneni PV: Treatment of cervical stenotic myelopathy: a cost and outcome comparison of laminoplasty versus laminectomy and lateral mass fusion. J Neurosurg Spine 14:619-625, 2011

6. Hirabayashi K, Watanabe K, Wakano K, Suzuki N, Satomi $\mathrm{K}$, Ishii Y: Expansive open-door laminoplasty for cervical spinal stenotic myelopathy. Spine (Phila Pa 1976) 8:693699, 1983

7. Hosono N, Yonenobu K, Ono K: Neck and shoulder pain after laminoplasty. A noticeable complication. Spine (Phila Pa 1976) 21:1969-1973, 1996

8. Karadimas SK, Erwin WM, Ely CG, Dettori JR, Fehlings MG: Pathophysiology and natural history of cervical spondylotic myelopathy. Spine (Phila Pa 1976) 38 (22 Suppl 1):S21-S36, 2013

9. Kuntz C IV, Levin LS, Ondra SL, Shaffrey CI, Morgan CJ: Neutral upright sagittal spinal alignment from the occiput to the pelvis in asymptomatic adults: a review and resynthesis of the literature. J Neurosurg Spine 6:104-112, 2007

10. Lee CH, Lee J, Kang JD, Hyun SJ, Kim KJ, Jahng TA, et al: Laminoplasty versus laminectomy and fusion for multilevel cervical myelopathy: a meta-analysis of clinical and radiological outcomes. J Neurosurg Spine 22:589-595, 2015

11. Lee DH, Park SA, Kim NH, Hwang CJ, Kim YT, Lee CS, et al: Laminar closure after classic Hirabayashi open-door laminoplasty. Spine (Phila Pa 1976) 36:E1634-E1640, 2011

12. Luo J, Cao K, Huang S, Li L, Yu T, Cao C, et al: Comparison of anterior approach versus posterior approach for the treatment of multilevel cervical spondylotic myelopathy. Eur Spine J 24:1621-1630, 2015

13. Manzano GR, Casella G, Wang MY, Vanni S, Levi AD: A 
prospective, randomized trial comparing expansile cervical laminoplasty and cervical laminectomy and fusion for multilevel cervical myelopathy. Neurosurgery 70:264-277, 2012

14. Matz PG, Anderson PA, Holly LT, Groff MW, Heary RF, Kaiser MG, et al: The natural history of cervical spondylotic myelopathy. J Neurosurg Spine 11:104-111, 2009

15. Núñez-Pereira S, Hitzl W, Bullmann V, Meier O, Koller H: Sagittal balance of the cervical spine: an analysis of occipitocervical and spinopelvic interdependence, with C-7 slope as a marker of cervical and spinopelvic alignment. J Neurosurg Spine 23:16-23, 2015

16. Nurboja B, Kachramanoglou C, Choi D: Cervical laminectomy vs laminoplasty: is there a difference in outcome and postoperative pain? Neurosurgery 70:965-970, 2012

17. Nurick S: The pathogenesis of the spinal cord disorder associated with cervical spondylosis. Brain 95:87-100, 1972

18. Protopsaltis T, Schwab F, Bronsard N, Smith JS, Klineberg $\mathrm{E}$, Mundis G, et al: The T1 pelvic angle, a novel radiographic measure of global sagittal deformity, accounts for both spinal inclination and pelvic tilt and correlates with health-related quality of life. J Bone Joint Surg Am 96:1631-1640, 2014

19. Sakaura H, Hosono N, Mukai Y, Iwasaki M, Yoshikawa H: Medium-term outcomes of C3-6 laminoplasty for cervical myelopathy: a prospective study with a minimum 5-year follow-up. Eur Spine J 20:928-933, 2011

20. Scheer JK, Tang JA, Smith JS, Acosta FL Jr, Protopsaltis TS, Blondel B, et al: Cervical spine alignment, sagittal deformity, and clinical implications: a review. J Neurosurg Spine 19:141-159, 2013

21. Schwab F, Patel A, Ungar B, Farcy JP, Lafage V: Adult spinal deformity-postoperative standing imbalance: how much can you tolerate? An overview of key parameters in assessing alignment and planning corrective surgery. Spine (Phila Pa 1976) 35:2224-2231, 2010

22. Subramaniam V, Chamberlain RH, Theodore N, Baek S, Safavi-Abbasi S, Senoğlu M, et al: Biomechanical effects of laminoplasty versus laminectomy: stenosis and stability. Spine (Phila Pa 1976) 34:E573-E578, 2009

23. Tang JA, Scheer JK, Smith JS, Deviren V, Bess S, Hart RA, et al: The impact of standing regional cervical sagittal alignment on outcomes in posterior cervical fusion surgery. Neurosurgery 76 (Suppl 1):S14-S21, 2015

24. Varthi AG, Basques BA, Bohl DD, Golinvaux NS, Grauer JN: Perioperative outcomes after cervical laminoplasty versus posterior decompression and fusion: analysis of 779 patients in the ACS-NSQIP database. J Spinal Disord Tech 29:E226-E232, 2016

25. Woods BI, Hohl J, Lee J, Donaldson W III, Kang J: Lamino- plasty versus laminectomy and fusion for multilevel cervical spondylotic myelopathy. Clin Orthop Relat Res 469:688695, 2011

26. Yamane K, Sugimoto Y, Tanaka M, Arataki S, Takigawa T, Ozaki T: Laminar closure rates in patients with cervical myelopathies treated with either open-door laminoplasty with reattachment of spinous processes and extensor musculature or Hirabayashi open-door laminoplasty: a case-control study. Eur Spine J 25:1869-1874, 2016

27. Yoon ST, Hashimoto RE, Raich A, Shaffrey CI, Rhee JM, Riew KD: Outcomes after laminoplasty compared with laminectomy and fusion in patients with cervical myelopathy: a systematic review. Spine (Phila Pa 1976) 38 (22 Suppl 1): S183-S194, 2013

\section{Disclosures}

Dr. Chou is a consultant for Medtronic and Globus and receives royalties from Globus. Dr. Mummaneni is a consultant for DePuy Spine and Stryker, has ownership in Spinicity/ISD, and has received royalties from DePuy Spine, Thieme Publishing, and Springer Publishing and honoraria from Globus and AOSpine, as well as a grant from AOSpine and ISSG. Dr. Than is a consultant for Bioventus.

\section{Author Contributions}

Conception and design: Mummaneni, Lau. Acquisition of data: Lau, Winkler, Chou. Analysis and interpretation of data: Mummaneni, Lau, Winkler. Drafting the article: Lau. Critically revising the article: all authors. Reviewed submitted version of manuscript: all authors. Approved the final version of the manuscript on behalf of all authors: Mummaneni. Statistical analysis: Lau. Study supervision: Mummaneni, Chou.

\section{Supplemental Information}

\section{Previous Presentations}

Portions of this work were presented at the AANS/CNS Joint Section on Disorders of the Spine and Peripheral Nerves, March 16-19, 2016, in Orlando, Florida.

\section{Correspondence}

Praveen V. Mummaneni, Department of Neurological Surgery, University of California, San Francisco, 505 Parnassus Ave., Rm. M779, San Francisco, CA 94143-0112. email: praveen. mummaneni@ucsf.edu. 